\title{
Generalized Residual Entropy and Upper Record Values
}

\author{
Suchandan Kayal \\ Department of Mathematics, National Institute of Technology Rourkela, Rourkela 769008, India \\ Correspondence should be addressed to Suchandan Kayal; suchandan.kayal@gmail.com
}

Received 1 June 2015; Accepted 26 July 2015

Academic Editor: Ramón M. Rodríguez-Dagnino

Copyright (C) 2015 Suchandan Kayal. This is an open access article distributed under the Creative Commons Attribution License, which permits unrestricted use, distribution, and reproduction in any medium, provided the original work is properly cited.

\begin{abstract}
In this communication, we deal with a generalized residual entropy of record values and weighted distributions. Some results on monotone behaviour of generalized residual entropy in record values are obtained. Upper and lower bounds are presented. Further, based on this measure, we study some comparison results between a random variable and its weighted version. Finally, we describe some estimation techniques to estimate the generalized residual entropy of a lifetime distribution.
\end{abstract}

\section{Introduction}

There have been several attempts made by various researchers to generalize the Shannon entropy (see Shannon [1]) since its appearance in Bell System Technical Journal. For various properties and applications of the generalized entropy measures, we refer to Kapur [2], Renyi [3], Tsallis [4], and Varma [5]. In this paper, we consider generalized residual entropy due to Varma [5]. Let $X$ be a nonnegative random variable representing the lifetime of a system with an absolutely continuous cumulative distribution function $F(x)$, probability density function $f(x)$, survival function $\bar{F}(x)(=1-F(x))$, and hazard rate $\lambda_{F}(x)$. The generalized entropy of $X$ is given by (see Varma [5])

$$
\begin{aligned}
& V_{\alpha, \beta}(X)=\left(\frac{1}{\beta-\alpha}\right) \ln \int_{0}^{\infty} f^{\alpha+\beta-1}(x) d x, \\
& 0 \leq \beta-1<\alpha<\beta .
\end{aligned}
$$

Measure (1) reduces to Renyi entropy (see Renyi [3]) when $\beta=1$ and reduces to Shannon entropy (see Shannon [1]) when $\beta=1$ and $\alpha \rightarrow 1$. We often find some situations in practice where the measure defined by (1) is not an appropriate tool to deal with uncertainty. For example, in reliability and life testing studies, sometimes it is required to modify the current age of a system. Here, one may be interested to study the uncertainty of the random variable $X_{t}=[X-t \mid X \geq t]$. The random variable $X_{t}$ is dubbed as the residual lifetime of a system which has survived up to time $t \geq 0$ and is still working. Analogous to Ebrahimi [6], the generalized entropy of the residual lifetime $X_{t}$ is given by

$$
\begin{aligned}
V_{\alpha, \beta}(X ; t)=\left(\frac{1}{\beta-\alpha}\right) \ln \int_{t}^{\infty} \frac{f^{\alpha+\beta-1}(x)}{\bar{F}^{\alpha+\beta-1}(t)} d x, \\
\\
\quad 0 \leq \beta-1<\alpha<\beta, t \geq 0,
\end{aligned}
$$

which is also known as the generalized residual entropy. It reduces to (1) when $t=0$. Also (2) reduces to Renyi's residual entropy (see Asadi et al. [7]) when $\beta=1$ and reduces to residual entropy (see Ebrahimi [6]) when $\beta=1$ and $\alpha \rightarrow 1$. Based on the generalized entropy measures given in (1) and (2), several authors obtained various results in the literature. In this direction, we refer to Kayal [8-11], Kayal and Vellaisamy [12], Kumar and Taneja [13], and Sati and Gupta [14]. In this paper, we study some properties and characterizations of the generalized residual entropy given by (2) based on the upper record values.

Let $\left\{X_{n}: n=1,2, \ldots\right\}$ be a sequence of identically and independently distributed nonnegative random variables having an absolutely continuous cumulative distribution function $F(x)$, probability density function $f(x)$, and survival function $\bar{F}(x)$. An observation $X_{j}$ in an infinite sequence $X_{1}, X_{2}, \ldots$ is said to be an upper record value if its value is greater than that of all the previous observations. For convenience, we denote $U_{1}=1$ and, for $i \geq 2, U_{i}=$ $\min \left\{j ; U_{i-1}<j\right.$ and $\left.X_{j}>X_{U_{i-1}}\right\}$. Then, $X_{U_{1}}, X_{U_{2}}, \ldots$ is called a sequence of upper record values. The probability 
density function and the survival function of the $n$th upper record value $X_{U_{n}}$ are given by

$$
\begin{aligned}
f_{U_{n}}(x) & =\frac{H^{n-1}(x)}{(n-1) !} f(x), \\
\bar{F}_{U_{n}}(x) & =\sum_{j=0}^{n-1} \frac{H^{j}(x)}{j !} \bar{F}(x)=\frac{\Gamma(n ; H(x))}{\Gamma(n)},
\end{aligned}
$$

respectively, where $H(x)=-\ln \bar{F}(x)$ and $\Gamma(a ; x)=$ $\int_{x}^{\infty} e^{-u} u^{a-1} d u, a>0, x \geq 0$. Note that $\Gamma(a ; x)$ is known as incomplete gamma function. Also the hazard rate of $X_{U_{n}}$ is

$$
\lambda_{F_{U_{n}}}(x)=\frac{H^{n-1}(x) /(n-1) !}{\sum_{j=0}^{n-1} H^{n}(x) / j !} \lambda_{F}(x) .
$$

Record values have wide spread applications in real life. For the applications of record values in destructive testing of wooden beams and industrial stress testing, one may refer to Glick [15] and Ahmadi and Arghami [16]. Record values are also useful in meteorological analysis and hydrology. For an extensive study of record values and applications, we refer to Arnold et al. [17]. The paper is arranged as follows.

In Section 2, we obtain various properties on the generalized residual entropy. It is shown that the measure given by (2) of the $n$th upper record value of any distribution can be expressed in terms of that of the $n$th upper record value from $U(0,1)$ distribution. Upper and lower bounds are obtained. Monotone behaviour of (2) based on the upper record values is investigated. In Section 3, based on (2), we study comparisons between a random variable and its weighted version. We describe some estimation techniques to estimate the generalized residual entropy of a life distribution in Section 4. Some concluding remarks have been added in Section 5. Throughout the paper, we assume that the random variables are nonnegative. The terms increasing and decreasing stand for nondecreasing and nonincreasing, respectively.

\section{Main Results}

In this section, we study several properties of the generalized residual entropy given by (2) based on the upper record values. First, we state the following lemma. The proof is straightforward hence omitted.

Lemma 1. Let $X_{U_{n}^{*}}$ denote the nth upper record value from a sequence of independent observations from $U(0,1)$. Then,

$$
\begin{aligned}
& V_{\alpha, \beta}\left(X_{U_{n}^{*}} ; t\right) \\
& \quad=\left(\frac{1}{\beta-\alpha}\right) \ln \frac{\Gamma(\gamma(n-1)+1,-\ln (1-t))}{\Gamma^{\gamma}(n ;-\ln (1-t))} .
\end{aligned}
$$

In the following theorem, we show that the generalized residual entropy of the upper record value $X_{U_{n}}$ can be expressed in terms of that of $X_{U_{n}^{*}}$. Let $X$ be a random variable having truncated gamma distribution with density function

$$
\begin{aligned}
f(x \mid a, b)=\frac{b^{a}}{\Gamma(a, t)} e^{-b x} x^{a-1}, & \\
x>t \geq 0, a>0, b & >0 .
\end{aligned}
$$

For convenience, we denote $X \sim \Gamma^{t}(a, b)$.

Theorem 2. The generalized residual entropy of $X_{U_{n}}$ can be expressed as

$$
\begin{aligned}
V_{\alpha, \beta} & \left(X_{U_{n}} ; t\right) \\
= & V_{\alpha, \beta}\left(X_{U_{n}^{*}} ; F(t)\right) \\
& \quad+\left(\frac{1}{\beta-\alpha}\right) \ln \left[E\left(f^{\gamma-1}\left(F^{-1}\left(1-e^{-V_{n}}\right)\right)\right)\right],
\end{aligned}
$$

where $V_{n} \sim \Gamma^{-\ln \bar{F}(t)}(\gamma(n-1)+1,1)$.

Proof. From (2), (3), and (4) and using the transformation $u=-\ln \bar{F}(x)$, we obtain

$$
\begin{aligned}
& V_{\alpha, \beta}\left(X_{U_{n}} ; t\right)=\left(\frac{1}{\beta-\alpha}\right) \\
& \cdot \ln \int_{-\ln \bar{F}(t)}^{\infty} \frac{f^{\gamma-1}\left(F^{-1}\left(1-e^{-u}\right)\right) e^{-u} u^{(n-1) \gamma}}{[\Gamma(n ;-\ln \bar{F}(t))]^{\gamma}} d u \\
& =V_{\alpha, \beta}\left(X_{U_{n}^{*}} ; F(t)\right)+\left(\frac{1}{\beta-\alpha}\right) \\
& \cdot \ln \left[E\left(f^{\gamma-1}\left(F^{-1}\left(1-e^{-V_{n}}\right)\right)\right)\right],
\end{aligned}
$$

since $V_{\alpha, \beta}\left(X_{U_{n}^{*}} ; F(t)\right)=\Gamma(\gamma(n-1)+1,-\ln \bar{F}(t))$. This completes the proof.

As a consequence of Theorem 2, we get the following remark.

Remark 3. Let $X_{U_{n}^{E}}$ denote the $n$th upper record value from a sequence of independent observations from a standard exponential distribution. Then,

$$
\begin{aligned}
V_{\alpha, \beta}\left(X_{U_{n}^{E}} ; t\right)= & \left(\frac{1}{\beta-\alpha}\right) \ln \frac{\Gamma(\gamma(n-1)+1, t)}{\Gamma^{\gamma}(n, t)} \\
& +\left(\frac{1}{\beta-\alpha}\right) \ln E\left(e^{-(\gamma-1) Z}\right),
\end{aligned}
$$

where $Z \sim \Gamma^{t}(\gamma(n-1)+1,1)$.

In Table 1, we obtain expressions of $V_{\alpha, \beta}\left(X_{U_{n}} ; t\right)$ for Weibull and Pareto distributions. It is easy to show that $\lambda_{F_{U_{n}}}(t) / \lambda_{F}(t)$ and $f_{U_{n}}(t) / f(t)$ are increasing functions in $t \geq$ 0 (see Li and Zhang [18]). Therefore, we have the following theorem whose proof follows along the lines similar to those in Theorem 8 of Kayal [11]. 
TABLE 1: Expressions of $V_{\alpha, \beta}\left(X_{U_{n}} ; t\right)$.

\begin{tabular}{lr}
\hline Probability density functions (PDF) & $V_{\alpha, \beta}\left(X_{U_{n}} ; t\right)$ \\
\hline$f(x \mid \theta)=\theta x^{\theta-1} e^{-x^{\theta}}, x>0, \theta>0$ & $\left(\frac{1}{\beta-\alpha}\right) \ln \frac{\Gamma\left(n \gamma-((\gamma-1) / \theta) ; t^{\theta}\right)}{\Gamma^{\gamma}\left(n ; t^{\theta}\right)}+\left(\frac{\gamma-1}{\beta-\alpha}\right) \ln \theta-\left(\frac{n \gamma-(1 / \theta)(\gamma-1)}{\beta-\alpha}\right) \ln \gamma$ \\
$f(x \mid \theta, \delta)=\frac{\theta \delta^{\theta}}{x^{\theta+1}}, x \geq \delta>0, \theta>0$ & $\left(\frac{1}{\beta-\alpha}\right) \ln \left(\frac{(\theta / \delta)^{\gamma-1} \Gamma(\gamma(n-1)+1 ;-\theta \ln (\delta / t))}{((1+(1 / \theta))(\gamma-1)+1)^{\gamma(n-1)+1} \Gamma^{\gamma}(n ;-\theta \ln (\delta / t))}\right)$ \\
\hline
\end{tabular}

TABLE 2: Bounds of $V_{\alpha, \beta}\left(X_{U_{n}} ; t\right)$.

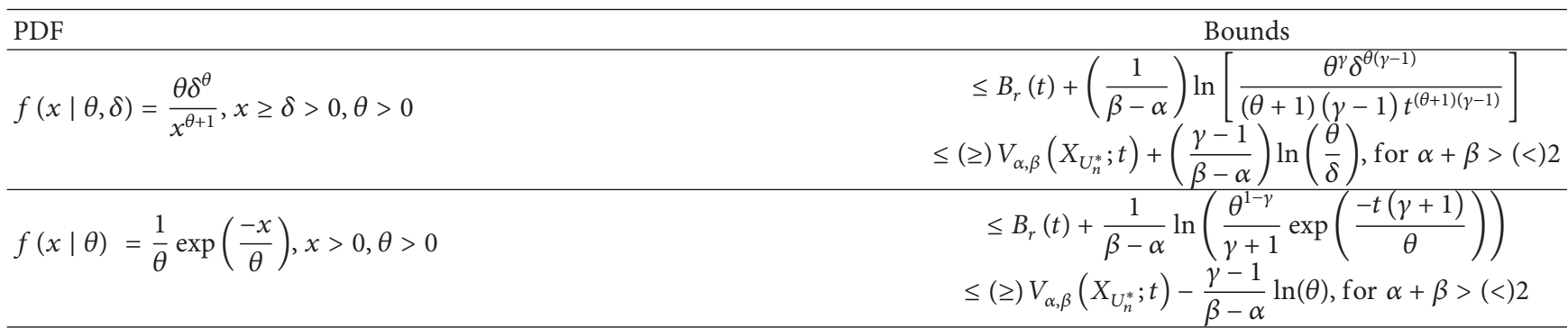

Theorem 4. The nth upper record value $X_{U_{n}}$ is increasing (decreasing) generalized residual entropy (IGRE (DGRE)) if $X$ is IGRE (DGRE).

Note that for $n$th and $(n+1)$ th upper record values, $\lambda_{F_{U_{n+1}}}(t) / \lambda_{F_{U_{n}}}(t)$ is increasing in $t \geq 0$ (see Kochar [19]) and hence $X_{U_{n}} \stackrel{l r}{\leq} X_{U_{n+1}}$. Therefore, the following corollary immediately follows from Theorem 4 .

Corollary 5. The $(n+1)$ th upper record value $X_{U_{n+1}}$ is IGRE (DGRE) if nth upper record value $X_{U_{n}}$ is IGRE (DGRE).

The following theorem provides bounds for the generalized residual entropy of the $n$th upper record $X_{U_{n}}$. We omit the proof as it follows in an analogous approach similar to that of Theorem 11 given in Kayal [11].

Theorem 6. (a) Let $V_{\alpha, \beta}\left(X_{U_{n}} ; t\right)$ be finite. If $m_{r}=\max \{\gamma(n-$ $1),-\ln \bar{F}(t)\}$ is the mode of $\Gamma^{-\ln \bar{F}(t)}(\gamma(n-1)+1,1)$, then

$$
\begin{aligned}
V_{\alpha, \beta}\left(X_{U_{n}} ; t\right) \leq & B_{r}(t) \\
& +\left(\frac{1}{\beta-\alpha}\right) \ln \int_{t}^{\infty} \lambda_{F}(x) f^{\gamma-1}(x) d x,
\end{aligned}
$$

where $B_{r}(t)=-(\gamma /(\beta-\alpha)) \ln \Gamma(n ;-\ln \bar{F}(t))+(\gamma(n-1) /(\beta-$ $\alpha)) \ln m_{r}-(1 /(\beta-\alpha)) m_{r}$.

(b) Let $M=f(m)<\infty$, where $m$ is the mode of the distribution with density function $f(x)$. Also $X_{U_{n}^{*}}$ denote the $n$th upper record value from a sequence of observation from $U(0,1)$. Then, for $\alpha+\beta>(<) 2$,

$$
V_{\alpha, \beta}\left(X_{U_{n}} ; t\right) \leq(\geq) V_{\alpha, \beta}\left(X_{U_{n}^{*}} ; t\right)+\left(\frac{\gamma-1}{\beta-\alpha}\right) \ln M .
$$

As an application of Theorem 6, we obtain bounds of Pareto and exponential distributions presented in Table 2. The following theorem gives the monotone behaviour of the generalized residual entropy of upper record values in terms of $n$.

Definition 7. Let $X$ and $Y$ be two nonnegative random variables with survival functions $\bar{F}(x)$ and $\bar{G}(x)$, respectively. Then, $X$ is said to be smaller than $Y$ in the usual stochastic ordering, denoted by $X \leq Y$, if $\bar{F}(x) \leq \bar{G}(x)$, for all $x \geq 0$.

Theorem 8. Let $X_{U_{1}}, X_{U_{2}}, \ldots$ be a sequence of upper record values from a distribution with cumulative distribution function $F(x)$ and probability density function $f(x)$. Also let $f(x)$ be an increasing function. Then, $V_{\alpha, \beta}\left(X_{U_{n}} ; t\right)$ is increasing (decreasing) in $n$ for $\alpha+\beta>(<) 2$.

Proof. From (8), we have

$$
\begin{aligned}
V_{\alpha, \beta}( & \left.X_{U_{n+1}} ; t\right)-V_{\alpha, \beta}\left(X_{U_{n}} ; t\right) \\
= & V_{\alpha, \beta}\left(X_{U_{n+1}^{*}} ; F(t)\right)-V_{\alpha, \beta}\left(X_{U_{n}^{*}} ; F(t)\right) \\
& +\left(\frac{1}{\beta-\alpha}\right) \ln \frac{E\left(f^{\gamma-1}\left(F^{-1}\left(1-e^{-V_{n+1}}\right)\right)\right)}{E\left(f^{\gamma-1}\left(F^{-1}\left(1-e^{-V_{n}}\right)\right)\right)} .
\end{aligned}
$$

Moreover, for $\alpha+\beta>(<) 2$,

$$
\frac{\int_{-\ln \bar{F}(t)}^{\infty} e^{-x} x^{\gamma(n-1)} d x}{\int_{-\ln \bar{F}(t)}^{\infty} e^{-x} x^{(n-1)} d x}
$$

is an increasing (decreasing) function in $t$. Therefore, for $u(x)=x$ and $v_{\gamma}(x)=e^{-x} x^{-\gamma}$, we have $\left.W_{\gamma}{ }^{\text {st }} \stackrel{\text { st }}{\leq}\right) W_{1}$ for $\alpha+\beta>(<) 2$. Hence, from Theorem 12 of Kayal [11], it can be proved that $V_{\alpha, \beta}\left(X_{U_{n}^{*}} ; F(t)\right)$ is increasing (decreasing) for $\alpha+$ $\beta>(<) 2$. Now, along the lines of the proof of Theorem 3.7 of Zarezadeh and Asadi [20], the proof follows. This completes the proof of the theorem. 


\section{Weighted Distributions}

To overcome the difficulty to model nonrandomized data set in environmental and ecological studies, Rao [21] introduced the concept of weighted distributions. Let the probability density function of $X$ be $f(x)$, and let $w(x)$ be the nonnegative function with $\mu_{w}=E(w(X))<\infty$. Also let $f_{w}(x)$ and $\bar{F}_{w}(x)$, respectively, be the probability density function and survival function of the weighted random variable $X_{w}$, which are given by

$$
\begin{aligned}
f_{w}(x) & =\frac{w(x) f(x)}{\mu_{w}}, \\
\bar{F}_{w}(x) & =\frac{E(w(X) \mid X \geq x) \bar{F}(x)}{\mu_{w}} .
\end{aligned}
$$

The hazard rate of $X_{w}$ is

$$
\lambda_{F_{w}}(x)=\frac{w(x)}{E(w(X) \mid X \geq x)} \lambda_{F}(x) .
$$

For some results and applications on weighted distributions, one may refer to Di Crescenzo and Longobardi [22], Gupta and Kirmani [23], Kayal [9], Maya and Sunoj [24], Navarro et al. [25], and Patil [26]. In the present section, we obtain some comparison results based on the generalized residual entropy between a random variable and its weighted version. We need the following definition in this direction.

Definition 9. A random variable $X$ with hazard rate $\lambda_{F}(x)$ is said to have a decreasing (increasing) failure rate (DFR (IFR)), if $\lambda_{F}(t)$ is decreasing (increasing) in $t \geq 0$.

Theorem 10. Let $X$ and $Y$ be two random variables with cumulative distribution functions $F(x)$ and $G(x)$, probability density functions $f(x)$ and $g(x)$, survival functions $\bar{F}(x)$ and $\bar{G}(x)$, and hazard rates $\lambda_{F}(x)$ and $\lambda_{G}(x)$, respectively. If $\lambda_{F}(t) \leq \lambda_{G}(t)$, for all $t \geq 0$, and either $F(x)$ or $G(x)$ is DFR, then $V_{\alpha, \beta}(X ; t) \leq(\geq) V_{\alpha, \beta}(Y ; t)$, for $\alpha+\beta>(<) 2$.

Proof. The proof follows along the lines of that of Theorem 4 of Asadi et al. [7].

Theorem 11. Under the assumptions of Theorem 10, if $\lambda_{F}(t) \geq$ $\lambda_{G}(t)$, for all $t \geq 0$, and either $F(x)$ or $G(x)$ is DFR, then $V_{\alpha, \beta}(X ; t) \geq(\leq) V_{\alpha, \beta}(Y ; t)$, for $\alpha+\beta>(<) 2$.

Proof. Proof follows from that of Theorem 4 of Asadi et al. [7].

Theorem 12. (a) Suppose $E(w(X) \mid X \geq t)$, or $w(t)$, is decreasing. If $X$ or $X_{w}$ is DFR, then, for all $t \geq 0, V_{\alpha, \beta}(X ; t) \leq$ $(\geq) V_{\alpha, \beta}\left(X_{w} ; t\right)$, for $\alpha+\beta>(<) 2$.

(b) Suppose $E(w(X) \mid X \geq t)$, or $w(t)$, is increasing. If $X$ or $X_{w}$ is DFR, then, for all $t \geq 0, V_{\alpha, \beta}(X ; t) \geq(\leq) V_{\alpha, \beta}\left(X_{w} ; t\right)$, for $\alpha+\beta>(<) 2$.

Proof. It is not difficult to see that $\lambda_{F}(t) \leq \lambda_{F_{w}}(t)$, for all $t \geq 0$, when either $E(w(X) \mid X \geq t)$ or $w(t)$ is decreasing. Now, the proof of part (a) follows from Theorem 10. Part (b) can be proved similarly. This completes the proof of the theorem.

Let $X$ be a random variable with density function $f(x)$ and cumulative distribution function $F(x)$. Also let $\mu_{x}=$ $E(X)>0$ be finite. Denote the length biased version of $X$ by $X_{L}$. Then, the probability density function of $X_{L}$ is given by

$$
f_{L}(x)=\frac{x f(x)}{\mu_{x}} .
$$

The random variable $X_{L}$ arises in the study of lifetime analysis and various probability proportional-to-size sampling properties. Associated with a random variable $X$, one can define another random variable $X_{E}$ with density function

$$
f_{E}(x)=\frac{\bar{F}(x)}{\mu_{x}} .
$$

This distribution is known as equilibrium distribution of $X$. The random variables $X_{L}$ and $X_{E}$ are weighted versions of $X$ with weight function $w_{L}(x)=x$ and $w_{E}(x)=1 / \lambda_{F}(x)$, respectively. The following corollary is a consequence of Theorem 12 .

Corollary 13. Let $X$ be DFR. Then, for all $t \geq 0$,
(a) $V_{\alpha, \beta}(X ; t) \geq(\leq) V_{\alpha, \beta}\left(X_{L} ; t\right)$ for $\alpha+\beta>(<) 2$;
(b) $V_{\alpha, \beta}(X ; t) \geq(\leq) V_{\alpha, \beta}\left(X_{E} ; t\right)$ for $\alpha+\beta>(<) 2$.

\section{Estimation}

In this section, we discuss the problem of estimation of the generalized residual entropy of a statistical distribution based on upper record values. Here, we consider exponential distribution. It has various applications in practice. Let $X$ follow exponential distribution with mean $\lambda$. Then, from (2), we obtain

$$
\begin{aligned}
V_{\alpha, \beta}(X ; t)= & \left(\frac{\alpha+\beta-1}{\beta-\alpha}\right) \ln \lambda \\
& -\left(\frac{1}{\beta-\alpha}\right) \ln (\alpha+\beta-1) .
\end{aligned}
$$

Based on the $n$ upper record values, the maximum likelihood estimator (mle) of $\lambda$ can be obtained as $\widehat{\delta}=X_{U_{n}} / n$, where $X_{U_{n}}$ is the $n$th upper record value. Now, applying invariance property, we obtain the mle of $V_{\alpha, \beta}(X ; t)$ as

$$
\begin{aligned}
\widehat{V}_{\alpha, \beta}^{\mathrm{ml}}= & \left(\frac{\alpha+\beta-1}{\beta-\alpha}\right) \ln \left(\frac{X_{U_{n}}}{n}\right) \\
& -\left(\frac{1}{\beta-\alpha}\right) \ln (\alpha+\beta-1) .
\end{aligned}
$$

Also the uniformly minimum variance unbiased estimator (umvue) of $V_{\alpha, \beta}(X ; t)$ can be obtained as 


$$
\begin{aligned}
\widehat{V}_{\alpha, \beta}^{\mathrm{mv}}= & \left(\frac{\alpha+\beta-1}{\beta-\alpha}\right) \ln \left(\frac{X_{U_{n}}}{\exp (\psi(n))}\right) \\
& -\left(\frac{1}{\beta-\alpha}\right) \ln (\alpha+\beta-1),
\end{aligned}
$$

where $\psi(n)$ is a digamma function. To illustrate the estimation techniques developed in this section, we consider simulated data from exponential distribution with mean 1 . In this purpose, we use Monte-Carlo simulation.

Example 14. In this example, we consider a simulated sample of size $n=5$ from the exponential distribution with mean 0.5. The simulated upper records are as follows:

$$
0.265410,0.637725,0.688878,0.791721 \text {, }
$$

$$
2.114831 \text {. }
$$

Based on these upper record values, we have $\widehat{V}_{\alpha, \beta}^{\mathrm{ml}}=-6.64759$ and $\widehat{V}_{\alpha, \beta}^{\mathrm{mv}}=-6.06166$ when $\alpha=1.2$ and $\beta=1.5$.

\section{Concluding Remarks}

In this paper, we consider generalized residual entropy due to Varma [5] of record values and weighted distributions. We obtain some results on monotone behaviour of this measure in upper record values. Some bounds are obtained. Further, some comparison results between a random variable and its weighted version based on the generalized residual entropy are studied. Finally, two estimators of the generalized residual entropy of exponential distribution have been described.

\section{Conflict of Interests}

The author declares that there is no conflict of interests regarding the publication of this paper.

\section{References}

[1] C. E. Shannon, "A mathematical theory of communication," The Bell System Technical Journal, vol. 27, no. 3, pp. 379-423, 1948.

[2] J. N. Kapur, "Generalized entropy of order alpha and type beta," in Proceedings of the Mathematical Seminar, pp. 78-94, New Delhi, India, 1967.

[3] A. Renyi, "On measures of entropy and information," in Proceedings of the 4th Berkeley Symposium on Mathematics, Statistics and Probability 1960, vol. 1, pp. 547-461, University of California Press, Berkeley, Calif, USA, 1961.

[4] C. Tsallis, "Possible generalization of Boltzmann-Gibbs statistics," Journal of Statistical Physics, vol. 52, no. 1-2, pp. 479-487, 1988.

[5] R. S. Varma, "Generalization of Renyi's entropy of order $\alpha$," Journal of Mathematical Sciences, vol. 1, pp. 34-48, 1966.

[6] N. Ebrahimi, "How to measure uncertainty in the residual life time distribution," Sankhyā, vol. 58, no. 1, pp. 48-57, 1996.

[7] M. Asadi, N. Ebrahimi, and E. S. Soofi, "Dynamic generalized information measures," Statistics \& Probability Letters, vol. 71, no. 1, pp. 85-98, 2005.
[8] S. Kayal, "On generalized dynamic survival and failure entropies of order $(\alpha, \beta)$," Statistics and Probability Letters, vol. 96, pp. 123132, 2015.

[9] S. Kayal, "Some results on dynamic discrimination measures of order $(\alpha, \beta)$," Hacettepe Journal of Mathematics and Statistics, vol. 44, pp. 179-188, 2015.

[10] S. Kayal, "Characterization based on generalized entropy of order statistics," Communications in Statistics-Theory and Methods. In press.

[11] S. Kayal, "Some results on a generalized residual entropy based on order statistics," Statistica. In press.

[12] S. Kayal and P. Vellaisamy, "Generalized entropy properties of records," Journal of Analysis, vol. 19, pp. 25-40, 2011.

[13] V. Kumar and H. C. Taneja, "Some characterization results on generalized cumulative residual entropy measure," Statistics \& Probability Letters, vol. 81, no. 8, pp. 1072-1077, 2011.

[14] M. M. Sati and N. Gupta, "On partial monotonic behaviour of Varma entropy and its application in coding theory," Journal of the Indian Statistical Association. In press.

[15] N. Glick, "Breaking records and breaking boards," The American Mathematical Monthly, vol. 85, no. 1, pp. 2-26, 1978.

[16] J. Ahmadi and N. R. Arghami, "Comparing the Fisher information in record values and iid observations," Statistics, vol. 37, no. 5, pp. 435-441, 2003.

[17] B. C. Arnold, N. Balakrishnan, and H. N. Nagaraja, Records, John Wiley \& Sons, New York, NY, USA, 1998.

[18] X. Li and S. Zhang, "Some new results on Rényi entropy of residual life and inactivity time," Probability in the Engineering and Informational Sciences, vol. 25, no. 2, pp. 237-250, 2011.

[19] S. C. Kochar, "Some partial ordering results on record values," Communications in Statistics-Theory and Methods, vol. 19, no. 1, pp. 299-306, 1990.

[20] S. Zarezadeh and M. Asadi, "Results on residual Renyi entropy of order statistics and record values," Information Sciences, vol. 180, no. 21, pp. 4195-4206, 2010.

[21] C. R. Rao, "On discrete distributions arising out of methods of ascertainment," Sankhyā, vol. 27, pp. 311-324, 1965.

[22] A. Di Crescenzo and M. Longobardi, "On weighted residual and past entropies," Scientiae Mathematicae Japonicae, vol. 64, no. 2, pp. 255-266, 2006.

[23] R. C. Gupta and S. N. U. A. Kirmani, "The role of weighted distributions in stochastic modeling," Communications in Statistics-Theory and Methods, vol. 19, no. 9, pp. 3147-3162, 1990.

[24] S. S. Maya and S. M. Sunoj, "Some dynamic generalized information measures in the context of weighted models," Statistica, vol. 68, no. 1, pp. 71-84 (2009), 2008.

[25] J. Navarro, Y. del Aguila, and J. M. Ruiz, "Characterizations through reliability measures from weighted distributions," Statistical Papers, vol. 42, no. 3, pp. 395-402, 2001.

[26] G. P. Patil, "Weighted distributions," in Encyclopedia of Environmetrics, A. H. El-Shaarawi and W. W. Piegorsch, Eds., John Wiley \& Sons, Chichester, UK, 2002. 


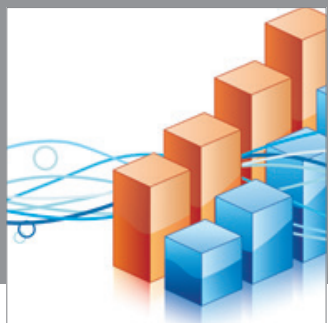

Advances in

Operations Research

mansans

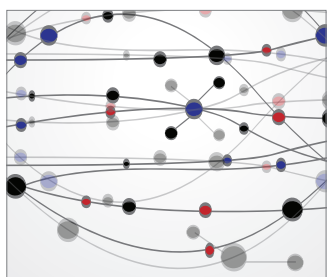

The Scientific World Journal
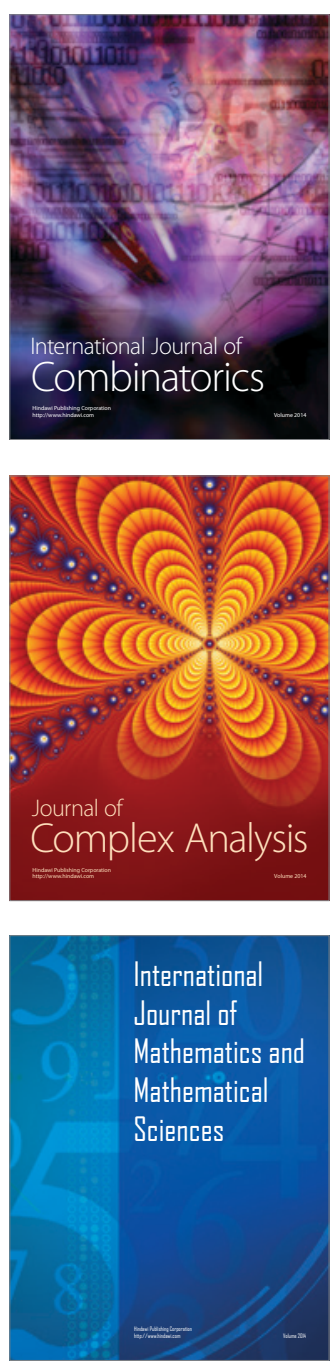
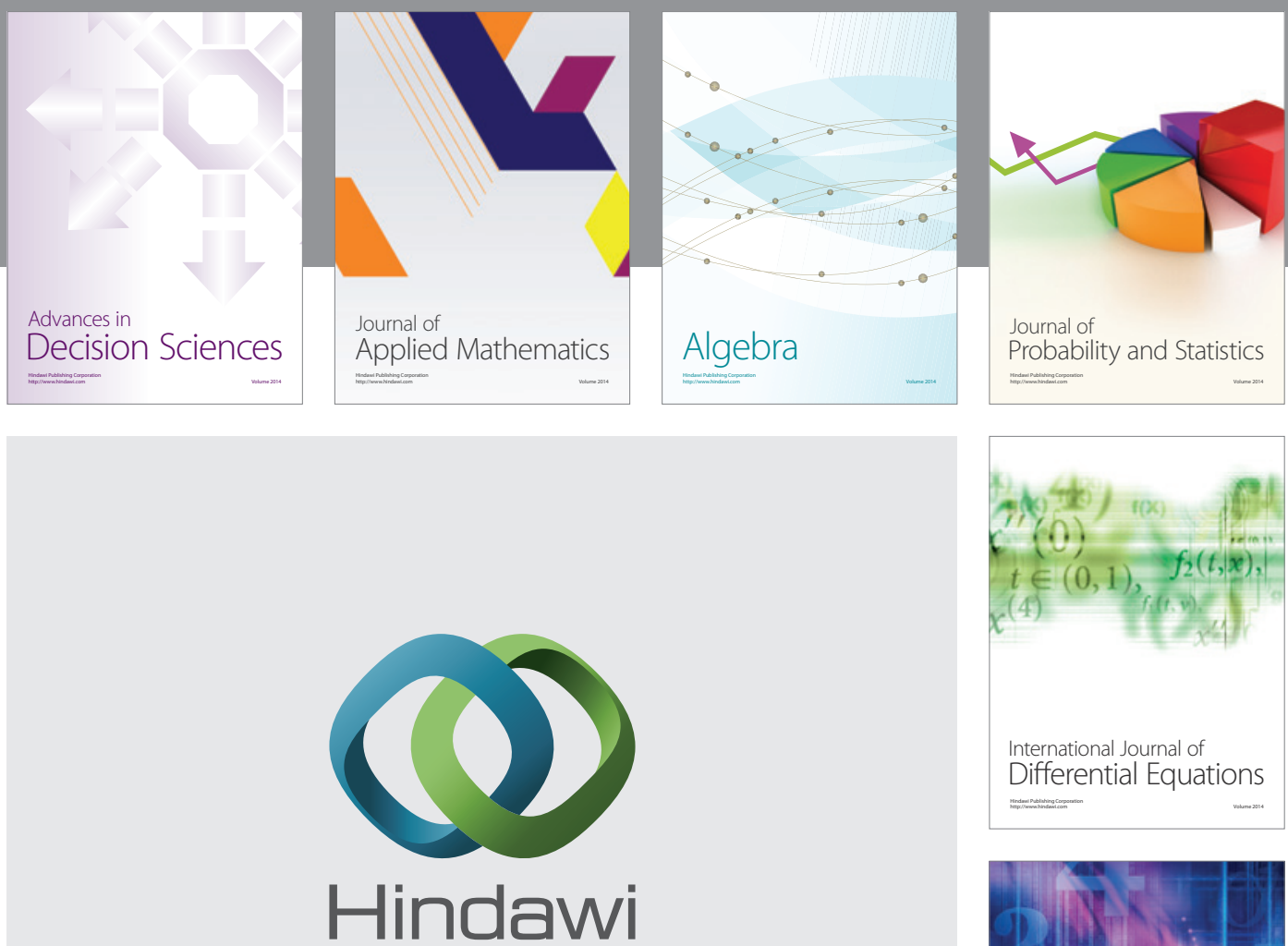

Submit your manuscripts at http://www.hindawi.com
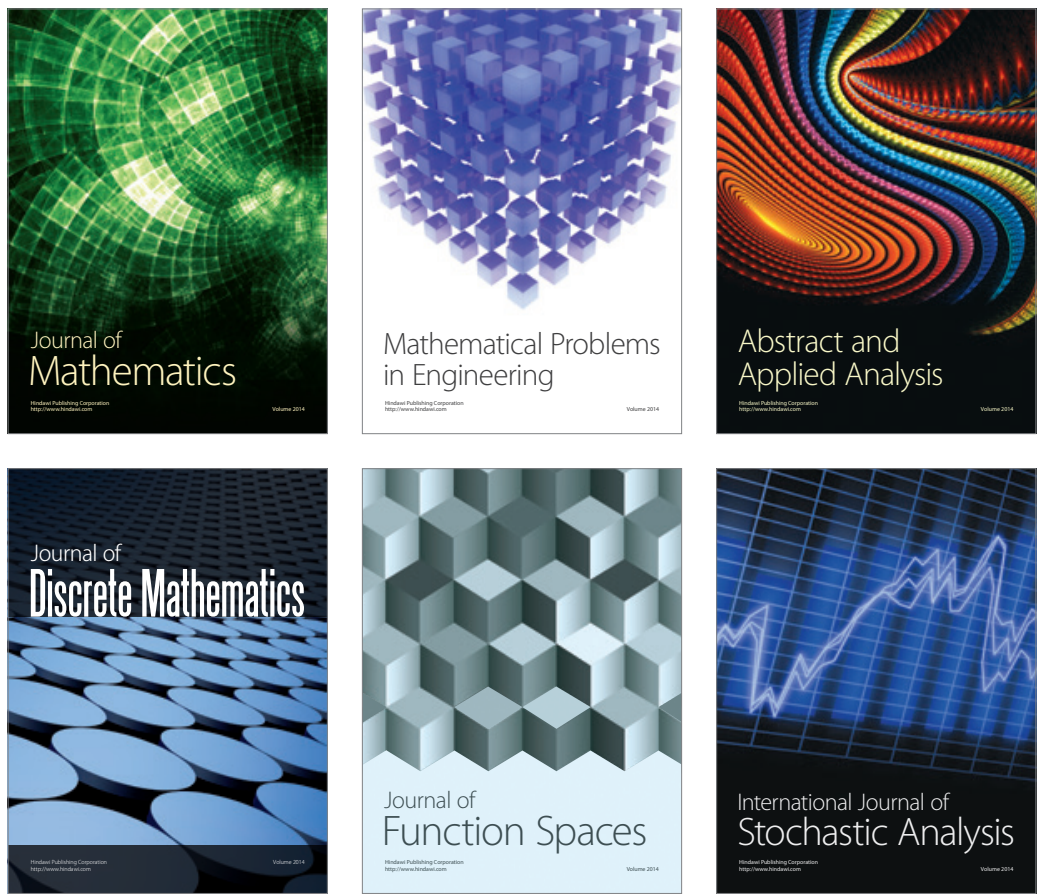

Journal of

Function Spaces

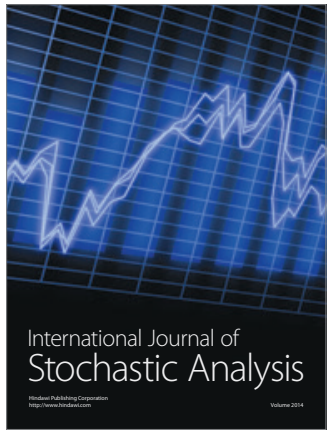

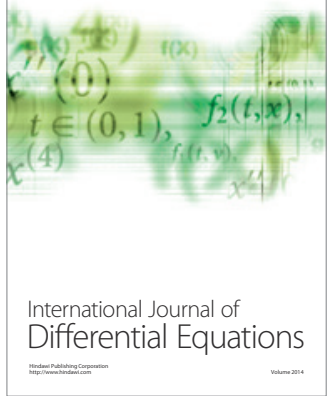
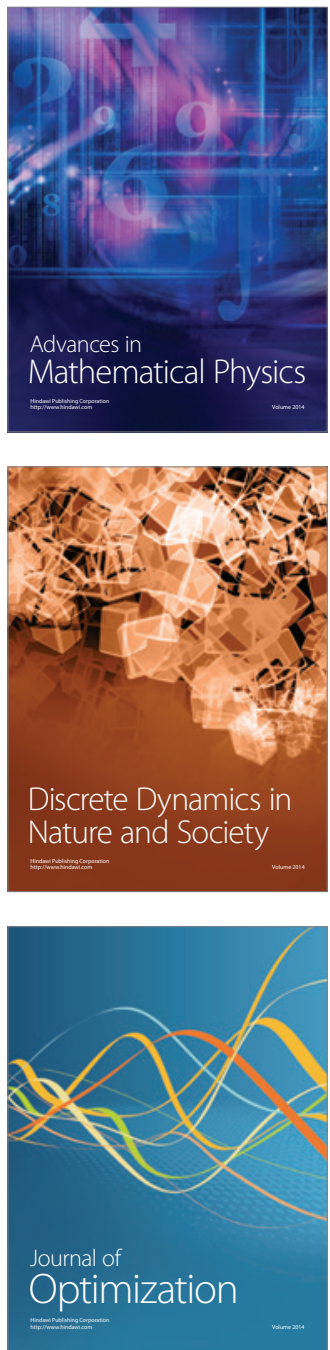